\title{
Limitations of Power-Spectrum and Time-Domain Analysis of Heart Rate Variability in Short-Term ECG Recorded using Telemetry in Unrestrained Rats
}

\author{
JP Couderc, A Elder, C Cox, W Zareba, G Oberdorster \\ University of Rochester, Rochester, NY, USA
}

\begin{abstract}
Radio-telemetry technique used to record electrophysiological signals in animals is broadly used in experimental studies. Using implantable probes, this technique can record electrocardiographic (ECG) signals without disturbing animal activities. In this study, we investigated the stability of heart rate variability $(H R V)$ parameters computed from radio-telemetry ECG recordings in unrestrained rats. The experiment involved 4 normal rats in which ECGs were recorded during baseline conditions with and without drug-induced autonomic blockade.

The study revealed that the stability of $H R V$ estimators for both time and frequency domains analysis in unrestrained rats was highly dependent on the average heart rate (related to physical rats activity) as well as the length of the ECG recording.
\end{abstract}

\section{Introduction}

There is growing interest in studying the autonomic nervous system regulation in animals exposed to molecule toxicity such as urban pollution [2]. Since autonomic nervous system (ANS) influences the heart rate by generating fluctuation in response to various stimuli from inside and outside of the body. The detection of abnormalities in the sympathovagal balance could contribute to better understand the link between pollution exposure and increased myocardial vulnerability [1].

In this study, we investigated the stability of time and frequency domain methods for estimating HRV from telemetric ECG signals. Specifically, we aimed to evaluate factors affecting the stability of the parameters used to estimate the HRV in unrestrained rats in order to guide future analyses using a similar experimental design. We investigated 4 healthy rats before, during and after autonomic blockade. We studied the values and the stability of time domain parameters including RMSSD and SDNN. The Fourier transformation was used to compute power spectral density of the tachogram and to extract the frequency bands of interest.

\section{Method}

\subsection{Animal experiment}

Four adult (6-8 months) specific pathogen-free male Fischer 344 rats (mean weight $357 \pm 27$ g) were obtained from Harlan (Indianapolis, IN). Rats were housed in wire-bottom cages and were allowed to acclimate for at least one week prior to use in experimental protocols.

Anaesthesia was induced with halothane (3\%; Halocarbon Laboratories, River Edge, NJ) and maintained throughout surgery $(0.75-1 \%)$. The body of the transmitter (TA11CTA-F40; Data Sciences International, St. Paul, MN) was placed in the abdominal cavity as suggested by the manufacturer. The ECG leads were placed under the skin and embedded in the muscles in a lead-II configuration. Rats were housed individually and allowed to recover from surgery for at least two weeks prior to any treatment.

ECGs were recorded using the Dataquest ${ }^{\mathrm{TM}}$ A.R.T. Gold System (Data Sciences International, St Paul, MN) which consisted of receivers placed under the cage of the rats that were connected to a personal computer (350 $\mathrm{MHz}$, Pentium II, $64 \mathrm{Mb}$ RAM) running under OS/2. The ECG signals were recorded with a sampling frequency of $1000 \mathrm{~Hz}$ and an amplitude resolution of 12 bits. All recordings were done in continuous mode.

\subsection{ECG recordings}

ECGs were recorded at baseline and after injection of atropine and propranolol. Atropine sulfate and DLpropranolol hydrochloride were purchased from Sigma Chemical Co. (St. Louis, MO) as dry powders. Solutions in sterile saline $(0.9 \%)$ were prepared on the day of each experiment. Due to the stress involved in tail vein injections, we chose to deliver these CNS-active agents via the intraperitoneal route $(5 \mathrm{mg} / \mathrm{kg})$.

ECGs were recorded continuously for up to 10 minutes. When baseline ECG recordings are obtained immediately prior to exposure for 30 minutes, this means that we have three continuous signals of 10 minutes. Postexposure recordings of the ECG potentials started 5 
minutes after injection of atropine or propranolol and continued for 30 minutes (again 3 times 10 minutes). Rats were unrestricted in their activity during recordings.

\subsection{HRV estimation}

Our methodology largely follows previous work $[3,4]$. We developed a software based on $\mathrm{C}++$ computer language extracting and interpolating the tachograms from the ECG signal. Frequency-domain parameters were computed from the tachogram after detrending in order to reduce contributions to the low-frequency power. The frequency-domain parameters were computed based on the power spectral density using Welch's averaged periodogram method. Based on previously published methods, the tachogram was interpolated and sampled every $130 \mathrm{~ms}(\Delta \mathrm{t})$. For every 10 minutes of the ECG period, the tachogram was divided into overlapping half sections of 512 samples $(\mathrm{N})$, each of which was windowed using a Hanning window (512-sample length). The frequency resolution was $\Delta \mathrm{f}=1 / \mathrm{N} \Delta \mathrm{t}=0.015 \mathrm{~Hz}$ and the maximum frequency was $3.85 \mathrm{~Hz}(\mathrm{~N} \Delta \mathrm{f} / 2)$.

We investigated two frequency-domain parameters. The energy of the high-frequency band (HF) between $0.7-2 \mathrm{~Hz}$ was defined by the integral of the power spectrum over this specific band. We used this band of frequencies to estimate the vagal control (parasympathetic tone). The low-frequency band was used to estimate sympathetic tone encompassing frequencies between 0.1 $0.7 \mathrm{~Hz}$. The frequency parameters are expressed in $\mathrm{ms}^{2} / \mathrm{Hz}$.

We implemented the two most common parameters for estimating HRV in the time-domain: SDNN and RMSSD. They are both expressed in milliseconds. SDNN is the standard deviation of the normalto-normal R-peak intervals and provides a measure of the total variability. RMSSD is the root mean square of successive differences. This last parameter quantifies the standard deviation of differences between neighboring intervals and estimates the parasympathetic drive of the ANS.

\subsection{Stability of HRV estimation}

Our primary objective was to investigate the stability of HRV estimation when ECGs are recorded using a telemetric system. The stability of RMSSD, SDNN, HF and LF parameters was studied. Using ECGs recorded during baseline (3 consecutive one-lead ECG strips of 10 minutes for each rats), the tachograms were extracted from each 10-minute ECG segments. Then, in order to assess stability of HRV parameters according to the number of beats, each tachogram was cut in sub-segments including an equal number of beats.

For instance the shorter subset included 128 beats. For an average RR values of $190 \mathrm{msec}$, each portion last approximately $24 \mathrm{sec}$. On 10 minutes tachogram, 25 consecutive non-overlapping segments of 128 beats are extracted. This decomposition is illustrated in Figure 1. We computed HRV estimators on the 24 segments and used the coefficient of variation (CVs) between segments for each parameters $X n$ such as $C V_{X}=\frac{\operatorname{std}(X)}{\bar{X}}$. Where $\mathrm{X}$ is a HRV parameter, std $(X)$ is the standard deviation from the $n$ values of estimator $\mathrm{X}(\mathrm{n}=24$ for 128 -beats segment) and $\bar{X}$ the mean values from the $n$ portions of the tachogram.

CVx were computed for all subset decompositions with a number of beats ranging from 128 to 2176 beats by step of 128 beats. The higher the CVs values the lower the stability and reproducibility of measurements.

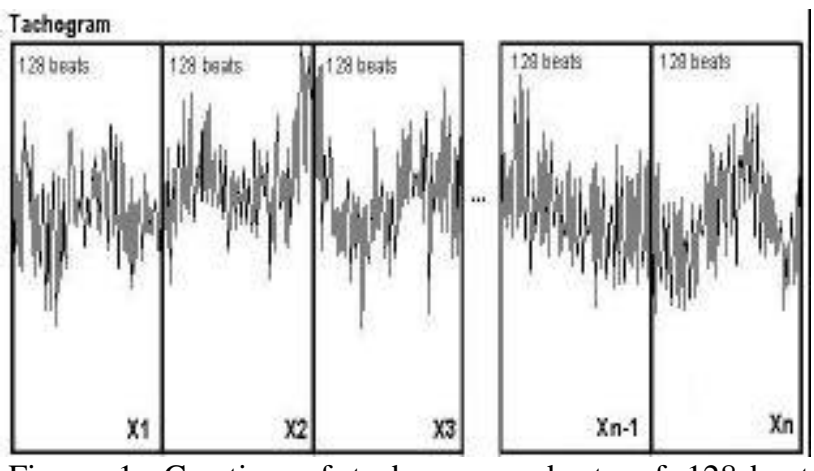

Figure 1. Creation of tachogram subsets of 128-beat duration.

\section{Results}

In Table 1, mean values of HRV parameters for baseline, under atropine and after propranolol injections are reported for all rats (periods 1 to 3). As expected, the results reveal a strong and significant autonomic blockade after atropine injection. SDNN is significantly decreased as well as HF frequency bands $(\mathrm{p}<0.05)$. Also the sympathetic tone was affected by atropine, we observed a significant decrease of LF components after atropine. After propranolol, no significant changes have been found, but in comparison to baseline, the autonomic blockade was still significantly reduced for both parasympathetic and sympathetic tones.

\subsection{HRV stability}

We studied the values of CVs in each rat separately. Figure 2 reports the changes in CVs between 128 and 2176 beats for time domain parameters whereas Figure 3 reports these changes for frequency-domain parameters. Obviously, we expect the values of CVs to decrease when the number of beats increases.

Table 1. Summary of the averaged values and standard 
deviations of all parameter values during baseline (1), under influence of atropine (2), under influence of propranolol and atropine (3). The values in bold are significantly different from baseline level with a $\mathrm{p}<0.05$. The values in italic are significantly different at a level of significance equal to 0.07 .

\begin{tabular}{cccccc}
\hline & $\begin{array}{c}\mathrm{HR} \\
\mathrm{bpm}\end{array}$ & $\begin{array}{c}\text { SDNN } \\
\mathrm{ms}\end{array}$ & $\begin{array}{c}\text { RMSSD } \\
\mathrm{Ms}\end{array}$ & $\begin{array}{c}\mathrm{HF} \\
\mathrm{ms}^{2} / \mathrm{Hz}\end{array}$ & $\begin{array}{c}\mathrm{LF} \\
\mathrm{ms}^{2} / \mathrm{Hz}\end{array}$ \\
\hline 1 & $320 \pm 7$ & $6.9 \pm 3.0$ & $2.3 \pm 1.3$ & $2.7 \pm 0.5$ & $4.9 \pm 1.1$ \\
2 & $\mathbf{4 7 0} \pm \mathbf{6}$ & $\mathbf{1 . 9} \pm \mathbf{0 . 1}$ & $1.0 \pm 0.4$ & $\mathbf{1 . 4} \pm \mathbf{0 . 3}$ & $\mathbf{2 . 4} \pm \mathbf{0 . 3}$ \\
3 & $\mathbf{3 8 0} \pm \mathbf{4}$ & $\mathbf{1 . 5} \pm \mathbf{0 . 2}$ & $0.8 \pm 0.3$ & $\mathbf{1 . 6} \pm \mathbf{0 . 2}$ & $\mathbf{2 . 6} \pm \mathbf{0 . 7}$ \\
\hline
\end{tabular}

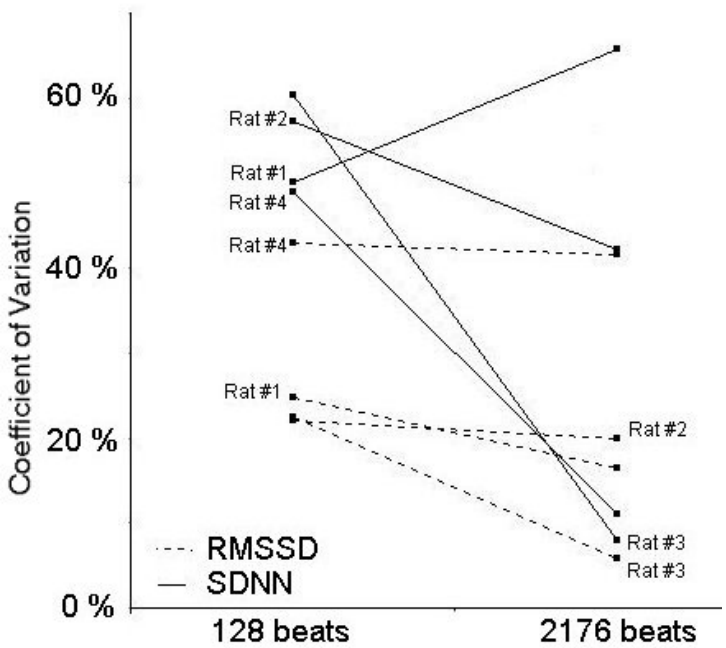

Figure 2. Changes in coefficients of variation from 128 to 2176 beats for RMSSD and SDNN parameters.

HRV parameters based on frequency domain were less stable than time-domain parameters regardless the number of beats included in the tachogram (Figure 3).

RMSSD and SDNN showed increased stability (lower $\mathrm{CVs}$ ) when the number of beats in the tachogram increases for rats \#3 and \#4. However, SDNN values for rat \#1 did not converge toward higher stability. Moreover, rat \#2 did show only a slight increased stability. In figure 4 , we report the evolution of the CVs for the SDNN parameter highlighting the unexpected evolution of stability for rat \#1.

Since all signals were of similar qualities and free of non-sinus beats, the difference between levels of stability across rats could only be explained by electrophysiological factors.

We computed the CVs for averaged heart rate, similarly as it was done for HRV parameters. These values were equal to 5.3, 2.1, 8.0 and $4.7 \%$ for rats \#1 to \#4, respectively. When we look at the curves in Figure 4, we see that the more stable measurements are obtained for rats \#3 above 1500 beats, this rat shows the highest stability of averaged HR (2.1\%). Rat \#4 is the following one showing a lack of stability around 2000 beats. Then, rats \#1 and \#2 reveal unreliable measurements of HRV.

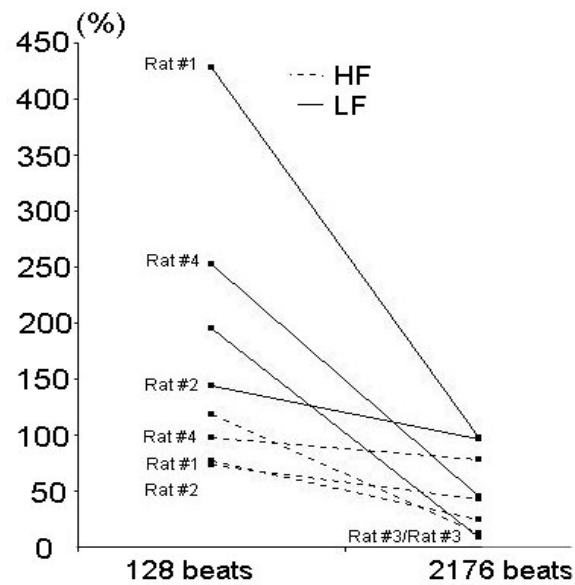

Figure 3. Changes in stability from 128 to 2176 beats for the HF and LF frequency bands.

Correlation analysis confirmed a significant association between the stability of average heart rate of tachogram and the stability of HRV estimators: $r=0.53$, $\mathrm{p}<0.001$ for SDNN; $\mathrm{r}=0.34, \mathrm{p}=0.003$ for RMSSD; $\mathrm{r}=0.60$, $\mathrm{p}<0.001$ for $\mathrm{LF}$ components and $\mathrm{r}=0.45, \mathrm{p}<0.001$ for HF components.

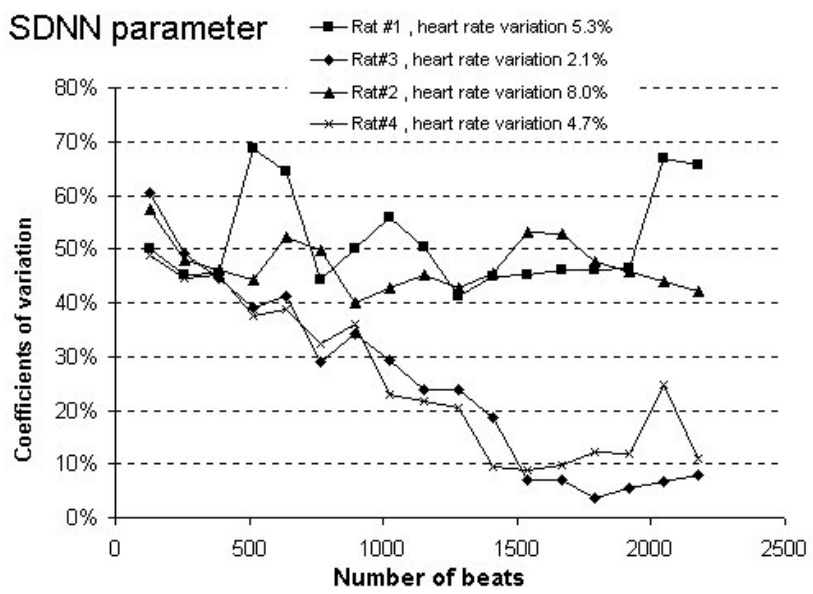

Figure 4. Evolution of the SDNN CVs according to the number of beats for each rat individually.

We conclude that two factors strongly affect the stability of HRV estimators when using time domain and non-parametric frequency approach: 1) average heart rate (considered as a surrogate of physical activity of the normal animals), 2) number of beats included in the tachogram.

\section{Discussion}

We investigated the stability of the frequency and time domain parameters when computed from ECGs of unrestrained rats using a telemetric system. 
This rat model and the telemetry equipment have been used to establish scientific basis of HRV parameters [3]. Nevertheless, there are very limited data regarding optimal methodology for HRV measurements in unrestrained animals. Moreover, external factors such social pressure, temperature, sounds have been shown to have an impact on the ANS regulation and thus affect experiment data.

The determination of frequency domain parameters based on the FFT transform approach has been very challenging, indeed the stability of HF and LF measurements on continuous data are non reproducible when rats are very active or show rapid changes in averaged heart rate. Our experiment thus reveals that doing HRV estimation in rats on short ECG recording $(<5$ minutes) requires a careful monitoring of the rat activities. According to our observations, one would recommend to estimate $\mathrm{HRV}$ while the animal has stable physical activities.

Sensitivity of the frequency method to animal activity can be explained by the lack of signal stationarity often reported as the major drawback of the nonparametric Fourier approach. Another component could be found in the complex elationship between HR and HRV, this relationship is not well understood but strong and physiological significant correlation, link to hemodynamic state, has been reported in humans between HR and HRV [5]. No study reported such relationship in rats where the heart rate can be up to 4 time higher with more rapid changes. More investigation is needed to further comment on this component at this point.

Using the Welch's averaged periodogram method as we did in the first part of the study partially corrects this lack of stability. However our recommendations following this preliminary study would be to use other approach like autoregressive approach or complex demodulation [5] when the ECG recording is short.

In conclusion, our study reveals strong limitations of HRV estimation from telemetric measurements of ECG signal in unrestrained rats. Time domain seems to be more stable than the non-parametric approach. Anyway, it is important to take the greatest care when estimating the HRV fluctuation from the power spectrum knowing since the method is sensitive to physiological factors impossible to control in unrestrained rats.

\section{Acknowledgements}

This research has been supported by grants (Particulate Matter Center grant R-827354-01-0 to GO and grant R82804601-0 to ACPE) from the U.S. Environmental Protection Agency's STAR program.

\section{References}

[1] Zareba W, Nomura A, Couderc JP. Cardiovascular effects of air pollution: What to measure in ECG? Environmental Health Perspectives 2001, Vol 109, Sup 4 ;533-538.

[2] Campen MJ, Watkinson WP, Nolan JP, Kodavanti UP, Evansky PA, Jenkins TP, Dowd SM, Costa DL. Effects of inhaled metallic constituents of residual oil fly ash particle on arrythmogenesis, electrocardiographic parameters, and heart rate variability in normal and compromised rats [Abstract]. Am J Respir Crit Care Med 161: A240 (2000).

[3] Akselrod S, Eliash S, Oz O, Cohen S. Hemodynamic regulation in SHR: investigation by spectral analysis. Am J Physiol 1987; 253(1 Pt 2):H176-H183.

[4] Cerutti S, Gustin M, Paultre C, Lo M, Julien C, Vincent M, Sassard J. Autonomic nervous system and cardiovascular variability in rats: a spectral analysis approach. Am J Physiol 1991;H1292H1299.

[5] Coumel P, Maison-Blanche P, Catuli D. Heart rate and heart rate variability. In Heart rate Variability, Malik M, Camm Aj Eds; Futura publisher 1995:207221.

[6] Shin SJ, Tapp WN, Reisman SS, Natelson BH. Assessment of autonomic regulation of heart rate variability by the method of complex demodulation. IEE Trans Biomed Eng 1989; 36(2):274-283.

Address for correspondence. Jean-Philippe Couderc, PhD 600 Elmwood Av. Box 653. 14642 Rochester, NY, USA

heartjpc@heart.rochester.edu 